\title{
GENERATION OF TUNABLE, MONOCHROMATIC X-RAYS IN THE LASER SYNCHROTRON SOURCE EXPERIMENT*
}

\author{
R.P. Fischer, A. Ting, C.I. Moore, and P. Sprangle \\ Plasma Physics Division, Naval Research Laboratory, Washington, DC \\ M. Baine, NASA Johnson Space Center, Houston, TX \\ R. Elton, University of Maryland, College Park, MD \\ S. Ride, University of California at San Diego, La Jolla, CA
}

\begin{abstract}
Tunable, monochromatic $\mathrm{x}$-rays have been generated in the Laser Synchrotron Source (LSS) experiment at the Naval Research Laboratory by Thomson backscattering of laser photons from a relativistic electron beam. Laser photons from a $5 \mathrm{~J}, 10 \mathrm{nsec}$, Nd:glass laser at $1053 \mathrm{~nm}$ wavelength are backscattered from a $4 \mathrm{MeV}, 300 \mathrm{~mA}$ electron beam generated by an S-band RF gun. Recent experimental results include the production of $10^{7} \mathrm{x}$-ray photons per pulse at $372 \mathrm{eV}$ with a bandwidth of $\pm 7 \mathrm{eV}$. Present work involves the characterization of the angular distribution of the x-rays and a new experiment to study nonlinear Thomson scattering using a 400 fsec, TW laser pulse.
\end{abstract}

\section{INTRODUCTION}

The development of compact, tunable, monochromatic $\mathrm{x}$-rays sources is important for advances in a number of fields including medical imaging, biology, solid state physics, and material sciences. In the LSS [1], the magnetic undulator of a conventional synchrotron is replaced with a laser undulator, which allows for the generation of hard x-rays using an electron beam with reduced energy. The goal of the present experiment is the production of $x$-rays in the water window (2-4 nm) using conventional laser and RF linac technology $[2,3]$.

\section{EXPERIMENTAL SET-UP}

A schematic diagram of the LSS experiment is shown in Figure 1 . The electron beam is generated by a 1 -and-1/2 cell RF gun powered by a $2.856 \mathrm{GHz}$ klystron with up to 10 MW. The thermionic emitter produces a $500 \mathrm{nsec}$ macropulse, which is comprised of a train of micropulses $10 \mathrm{psec}$ in duration separated by $350 \mathrm{psec}$. Electrons are emitted during all phases of the RF cycle, which results in a beam with relatively large energy spread. An energy selector is placed after the first bending magnet, so that only the most energetic electrons are passed down the beamline (1-2\% energy spread). After the second bending magnet (which corrects for chromatic aberration), the beam is focused at the interaction region using a set of quadrupole magnets and 2 steering magnets. Several Faraday cups are located along the beamline to measure the current, while phosphor screens and CCD cameras are used to monitor the beam profile. Electron beam size and centering are particularly important at the exit of the second bending magnet and the entrance of the beam dump. Electrons that strike the beam tube in these regions generate hard $\mathrm{x}$-rays in the line of sight of the detector, resulting in spurious $\mathrm{X}$-ray signals.

Table 1: Electron beam parameters

\begin{tabular}{|l|l|}
\hline Beam energy & $4.1 \mathrm{MeV}$ \\
\hline Macropulse current & $100-300 \mathrm{~mA}$ \\
\hline Radius at focus & $0.8 \mathrm{~mm}$ \\
\hline Energy spread & $1-2 \%$ \\
\hline Emittance & $9 \pi \mathrm{mm} \mathrm{mrad}$ \\
\hline Macropulse length & $250 \mathrm{nsec}$ \\
\hline Micropulse current & $3-10 \mathrm{~A}$ \\
\hline Micropulse length & $10 \mathrm{psec}$ \\
\hline Micropulse separation & $350 \mathrm{psec}$ \\
\hline
\end{tabular}

The LSS laser consists of a flashlamp-pumped, Qswitched, cavity-dumped Nd:YLF oscillator, a doublepassed $9 \mathrm{~mm}$ diameter $\mathrm{Nd}$ :Glass rod amplifier, and a double-passed $32 \mathrm{~mm}$ diameter Nd:Glass rod final amplifier. A Pockels cell after the oscillator is used as a pulse slicer to shorten the pulse length to $10 \mathrm{nsec}$. The laser is capable of producing 10-20 J of energy, although only $5 \mathrm{~J}$ are used in the present experiment due to the damage threshold of the optics in vacuum. A lens with a $2 \mathrm{~m}$ focal length is placed immediately before the vacuum window into the beamline, which focuses the laser to a radius of $220 \mu \mathrm{m}$ at the interaction region. The laser is then directed through the output vacuum window and onto a calorimeter to measure the energy. If the laser energy is too high, hot spots in the beam will damage one of the vacuum windows or the final turning mirror. Breakdown on the damaged optic generates visible and uv light, which results in spurious signals on the x-ray detector.

* Sponsored by ONR and the Medical FEL program. 


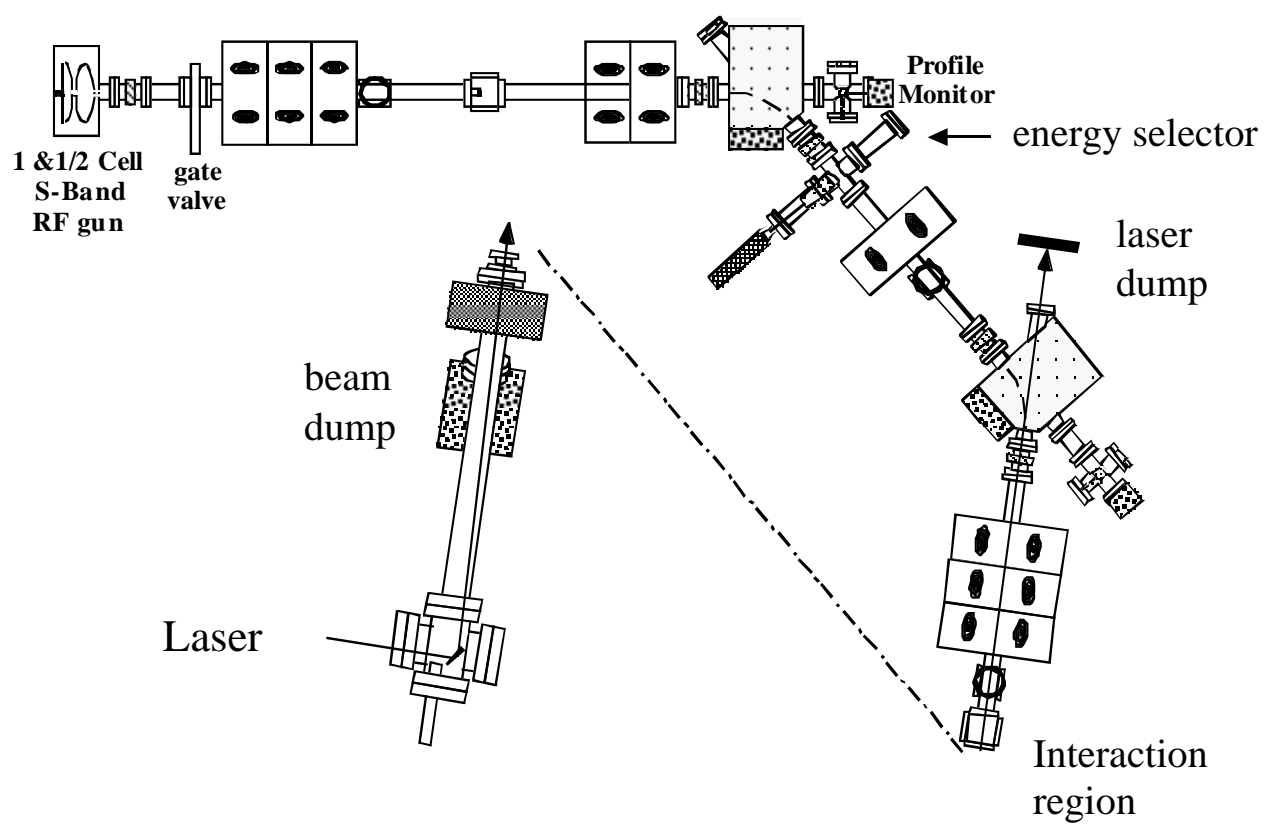

Figure 1: Schematic diagram of the LSS experiment

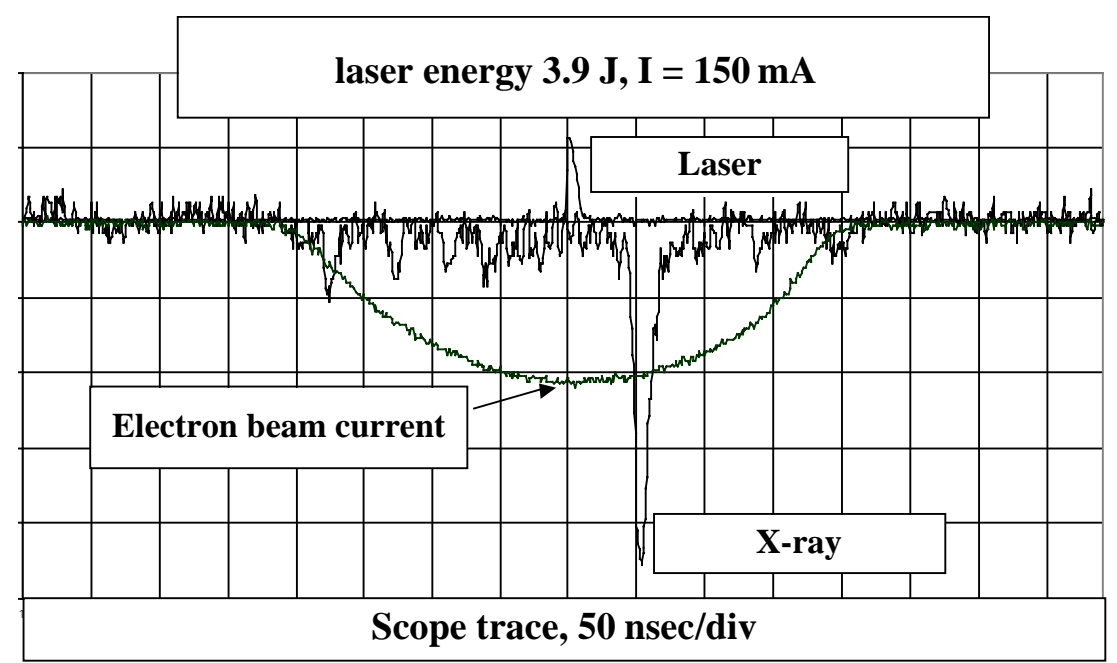

Figure 2: Oscilloscope trace showing the electron beam current, the laser pulse, and the detected x-ray signal.

The selection of the proper x-ray detector is crucial in the LSS experiment. The detector should be sensitive to the backscattered $400 \mathrm{eV}$ photons of interest, but should be insensitive to the hard $\mathrm{x}$-rays generated by the electron beam and any visible/uv light generated by the laser. Initially a photomultiplier tube (PMT), scintillator, and visible-light filter were used, but the PMT was too sensitive to the hard $\mathrm{x}$-rays and the visible-light leakage. An electron multiplier tube (EMT) proved to be a good choice of detector, which results in a low noise background during the $250 \mathrm{nsec}$ electron beam macropulse. The EMT has a $5 \mathrm{nsec}$ risetime, an internal delay of $40 \mathrm{nsec}$, and a gain of approximately $10^{6}$. The EMT is comprised of $21 \mathrm{Al}_{2} \mathrm{O}_{3}$ dynode stages biased with 2200 Volt dc.
Table 2: LSS laser parameters

\begin{tabular}{|l|l|}
\hline Laser energy & $5 \mathrm{~J}$ \\
\hline Pulse length & $10 \mathrm{nsec}$ \\
\hline Wavelength & $1.053 \mu \mathrm{m}$ \\
\hline Radius at focus & $220 \mu \mathrm{m}$ \\
\hline
\end{tabular}




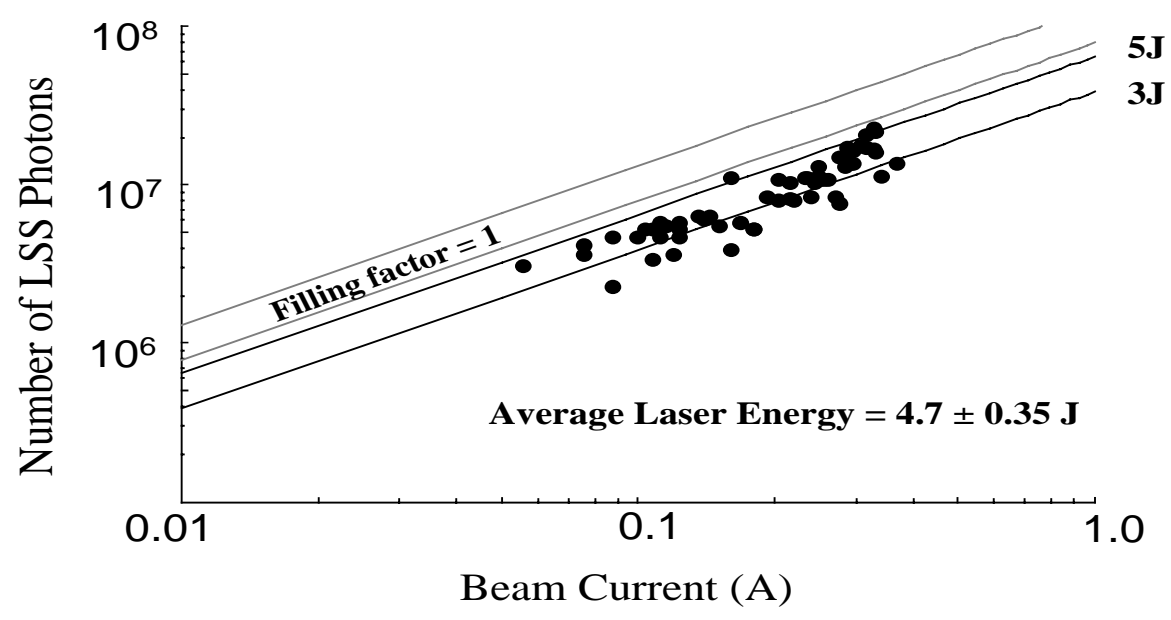

Figure 3: Number of LSS photons versus electron beam current.

\section{EXPERIMENTAL RESULTS}

A typical oscilloscope trace from the experiment is shown in Figure 2. The 250 nsec electron beam current pulse is measured using a Faraday cup in the beam dump. A photodiode is placed immediately before the input vacuum window, and serves as a timing fiducial. The $\mathrm{x}-$ ray signal in Figure 2 is detected $45 \mathrm{nsec}$ after the laser signal. This delay is the sum of the internal delay of the EMT, the time for the laser to propagate from the input window to the interaction region, the time for the $\mathrm{x}$-rays to propagate from the interaction region to the detector, and any differences in cable lengths.

Several null tests are performed which demonstrate that the x-ray signal is due only to the interaction of the laser pulse with the electron beam. When the laser or the electron beam is fired individually, no $x$-ray signal is observed. If the laser is fired before or after the electron beam, no $\mathrm{x}$-ray pulse is detected. A reduced $\mathrm{x}$-ray signal occurs if the laser is fired during the rise or fall of the electron current pulse. The relative timing between the laser and EMT signals is an important factor in discriminating between the desired LSS radiation and spurious $\mathrm{x}$-ray signals. If a laser arc occurs on the input window or final turning mirror, an EMT signal is observed 32 nsec after the laser fiducial. Likewise, a breakdown on the output window generates an EMT pulse with a delay of $52 \mathrm{nsec}$.

Figure 3 is a plot of the number of LSS photons versus the electron beam current. Approximately $1.5 \times 10^{7} \mathrm{x}$-ray photons are generated per pulse using $300 \mathrm{~mA}$ of electron beam current. The top two theory curves correspond to 3 and $5 \mathrm{~J}$ of laser energy, where it is assumed there is a perfect overlap of the laser spot and the electron beam (filling factor $=1$ ). The two lower theory curves are calculated using the measured spot sizes for the laser and electron beams and integrating over the interaction region. Good agreement is obtained between the theory and the measured data. The EMT was calibrated using an absolutely calibrated x-ray photodiode and a carbonanode bremsstrahlung $\mathrm{x}$-ray source.

The mean photon energy and bandwidth are measured by using a series of $\mathrm{x}$-ray filters with different thickness. These filters include $0.1 \mu \mathrm{m}$ titanium, $0.1 \mu \mathrm{m}$ silver, 0.1 $\mu \mathrm{m}$ silicon, and 0.5 and $1 \mu \mathrm{m}$ beryllium. The measured $\mathrm{x}$ ray data is compared to the theory using a least-square fitting analysis. The best fit is obtained for a photon energy of $372 \mathrm{eV}$ and bandwidth of $\pm 7 \mathrm{eV}$, which is quite close to the theoretical value of $375 \mathrm{eV}$.

\section{SUMMARY}

An LSS experiment has been performed which generates backscattered $\mathrm{x}$-ray photons from the interaction of a $5 \mathrm{~J}, 10 \mathrm{nsec}$ laser pulse with a $4 \mathrm{MeV}, 300 \mathrm{~mA}$ electron beam. The measured $\mathrm{x}$-rays characteristics are $10^{7}$ photons per pulse with energy $372 \mathrm{eV}$ and bandwidth $\pm 7 \mathrm{eV}$, which are in good agreement with theory. A new experiment is underway to measure the angular distribution of the backscattered x-ray photons by mounting the EMT on a translation stage in the vacuum chamber. Work is also continuing on new experiments to study nonlinear Thomson scattering using a $400 \mathrm{fsec}$, TW laser pulse.

\section{REFERENCES}

[1] P. Sprangle, A. Ting, E. Esarey, and A. Fisher, J. Appl. Phys. 72 (11), 1 Dec. 1992.

[2] A. Ting, R. Fischer, A. Fisher, C.I. Moore, B. Hafizi, R. Elton, K. Krushelnick, R. Burris, S. Jackel, K. Evans, J.N. Weaver, P. Sprangle, E. Esarey, M. Baine and S.K. Ride, Nucl. Instr. Meth. Phys. Res., A 375, ABS68 (1996).

[3] M. Baine, Ph.D. Thesis, University of California, San Diego, 2000. 